\title{
Large Morgagni Hernia in an Adult Patient
}

\author{
Sofia Marques Santos, Eunice Oliveira, Nuno Ferreira Monteiro, Maria Manuela Soares, Alberto Mello e Silva \\ Department of Internal Medicine, Egas Moniz Hospital, Lisbon, Portugal
}

Doi: 10.12890/2015_000322 - European Journal of Case Reports in Internal Medicine - @ EFIM 2015

\begin{abstract}
Received: 07/11/2015
\end{abstract}
Accepted: $12 / 12 / 2015$

Published: $21 / 12 / 2015$

How to cite this article: Marques Santos S, Oliveira E, Ferreira Monteiro N, Soares MM, Mello e Silva A. Large Morgagni hernia in an adult patient. EJCRIM 2015;2:doi:10.12890/2015_000322

Conflicts of Interests: The authors declare that there are no competing interests.

This article is licensed under a Commons Attribution Non-Commercial 4.0 License

\section{ABSTRACT}

A 70-year-old woman presented to the emergency department with symptoms of a lower respiratory infection. A chest $\mathrm{x}$-ray showed enlargement of the mediastinal space. The patient was admitted with a respiratory tract infection and started on antibiotic treatment. $A$ computed tomography (CT) scan of the thorax revealed a large diaphragmatic hernia with stomach, large intestine and caudal pancreas lodged in the thoracic cavity. After the antibiotic treatment, the patient became asymptomatic and surgery repair was declined.

Morgagni hernia is an uncommon type of congenital diaphragmatic hernia, which may be asymptomatic until late in life or may be present acutely with life threatening conditions.

\section{LEARNING POINTS}

- Morgagni hernia is the most rare form of congenital hernia, representing 2 to $3 \%$ of all cases.

- Chronic respiratory symptoms or gastrointestinal disturbances can be the only manifestation.

- Most asymptomatic cases in adults are found after doing chest x-ray for unrelated problems.

\section{KEYWORDS}

Morgagni hernia; diaphragmatic hernia.

\section{INTRODUCTION}

The Morgagni hernia consists of a defect in the anterior diaphragm, being more common on the right and allowing herniation of abdominal contents to the thorax ${ }^{1,2}$. It is the rarest form of congenital hernia, representing 2 to $3 \%$ of all cases ${ }^{2}$. Most adults present with non-specific respiratory and gastrointestinal symptoms ${ }^{2}$.

\section{CASE REPORT}

A 70-year-old woman, with a known history of hypertension, presented to the emergency department with purulent cough and dyspnoea, which had started the day before. She was hemodynamically stable but febrile $\left(38.8^{\circ} \mathrm{C}\right)$, with reduced breath sounds on the lower-right hemithorax. She had a slightly elevated $\mathrm{C}$ reactive protein $(5.5 \mathrm{mg} / \mathrm{dL})$ and hypoxemia in air room (pO2 $59 \mathrm{mmHg})$. Chest x-ray showed enlargement of the mediastinal space, with right-sided air-fluid level (Fig. 1), best visible in the right-lateral view (Fig. 2). She was initially admitted with the diagnosis of respiratory tract infection and empirically started on amoxicillin/clavulanate and clarithromycin (covering the most frequent respiratory pathogens from the community). The patient was then transferred to the medicine department and, upon arrival, it was decided to perform a computed tomography (CT) scan of the thorax to clarify the changes seen in the initial chest radiograph. 
The CT scan showed a massive supradiaphragmatic hernia, with interposition of bowel loops between the aortic arch and the cardiac silhouette (Fig. 3). There was a right-lower lobe collapse determined by this hernia, and there was also herniation of the entire stomach and part of the pancreas tail (Fig. 4) that was responsible for the opacity seen in the x-ray. The diagnosis of Morgagni hernia was made, with a concomitant lower respiratory tract infection. At the second day of therapy, the patient was afebrile and at the end of antibiotic treatment she was completely asymptomatic. The patient was also evaluated by a surgical team but she declined surgical correction.

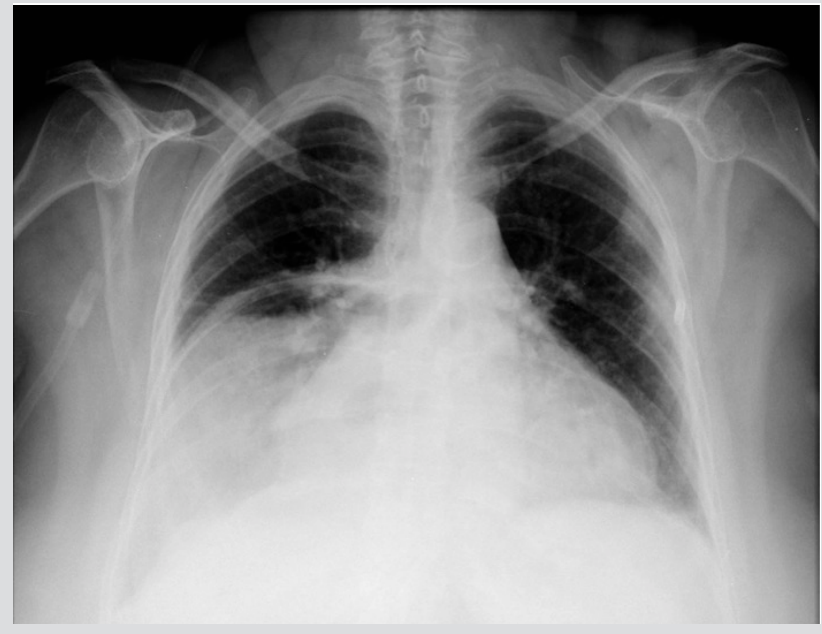

Figure 1. Chest $x$-ray in anterior view. Note the large right paracardiac opacity.

Figure 2. Chest $x$-ray in lateral view. Note the air-fluid levels present.
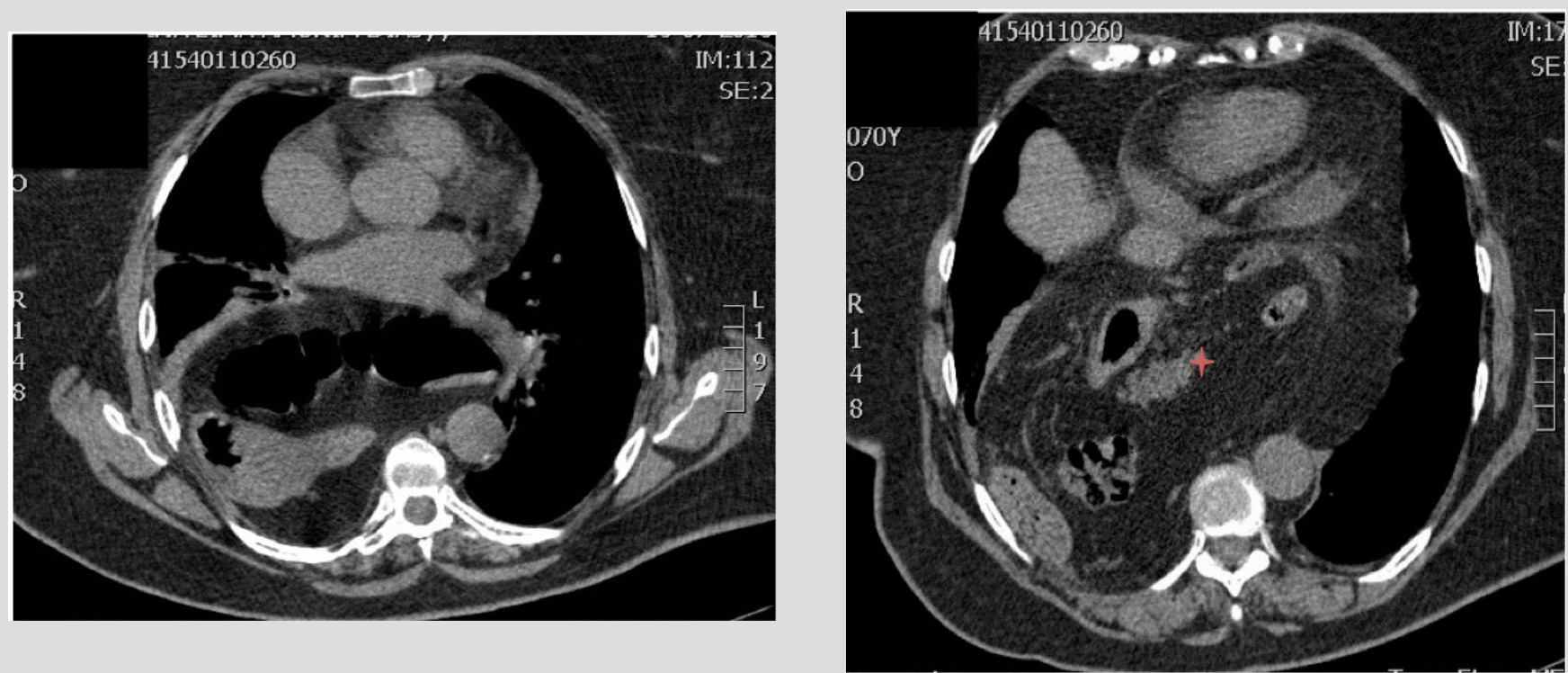

Figure 3. CT scan of the thorax, axial view, with interposition of bowel loops between the aortic arch and the cardiac silhouette.

Figure 4. CT scan of the thorax, axial view, with herniation of the stomach and part of the pancreas (red star). 


\section{DISCUSSION}

Morgagni hernias develop due to the lack of fusion of the sternal and crural portions of the diaphragm ${ }^{3}$. The most common viscus to herniate is the transverse colon, but it may also involve the liver, mesentery or, rarely, the stomach ${ }^{3}$. In this case, there was also the involvement of the caudal portion of the pancreas, which has seldom been seen. The presence of this hernia in adulthood is very rare and, when symptomatic, it is usually associated with chronic respiratory symptoms (dyspnoea, fatigue, pain) or gastrointestinal involvement with occlusive symptoms (constipation, occlusion) 4 . It may sometimes present with life-threatening conditions, such as bowel incarceration or peritonitis, being the diagnosis often delayed due to changes in anatomical relationship ${ }^{3}$. However, most patients remain asymptomatic and the majority of cases are discovered incidentally while investigating unrelated problems (like, for example, after performing a chest $\mathrm{x}$-ray) ${ }^{2}$. Depending on the contents of the hernia, the images in the chest radiography may appear differently ${ }^{2}$. Most frequently, it will show a solid paracardiac shadow (due to the presence of omentum) and the differential diagnosis should include a pleuropericardial cyst, lipoma, liposarcoma, mesothelioma, pericardial fat mass, diaphragmatic cyst and thymoma ${ }^{2,5}$.

If the presence of a Morgagni hernia is suspected, CT scan is considered to be an accurate and non-invasive form of diagnosis ${ }^{2}$. Another diagnostic option would be a barium enema, having the advantage of being less expensive ${ }^{2}$. When all imaging investigations are nondiagnostic, laparoscopic confirmation may be required ${ }^{2}$.

Although the majority of patients is asymptomatic, repair is almost always recommended to avoid future complications, especially when the colon is herniated due to the risk of obstruction ${ }^{3}$. On the other hand, if it contains only omentum, surgery is only indicated in the presence of symptoms ${ }^{2}$. This patient had a large amount of intestine in the thorax and had a full indication for surgical repair. Surgical treatment options include a transabdominal (laparotomy or laparoscopic) or transthoracic (video-assisted thoracoscopy) repair ${ }^{2,4}$. Follow-up after operative repair can be done with chest $x$-ray at three months and one year².

\section{REFERENCES}

1. Arora S, Haji A. Adult Morgagni hernia: the need for clinical awareness, early diagnosis and prompt surgical intervention. Ann R Coll Surg Eng 2008; 90:694-5.

2. Loong T, Kocher H. Clinical presentation and operative repair of hernia of Morgagni. Postgrad Med J 2005;81:41-4.

3. Daneshvar S, Shriki J, Sohn H, Rahimtoola SH. Morgagni-type diaphragmatic hernia presenting as an abnormal cardiac silhouette. Am J Med 2010;123:e11-2.

4. Marinceu D, Loubani M, O'Grady H. Late presentation of a large Morgagni hernia in an adult. BMJ Case Rep 2014;doi: 10.1136/bcr-2013-202440.

5. Komatsu T, Takahashi Y. Is this a mediastinal tumor? A case of Morgagni hernia complicated with intestinal incarceration mistaken for the mediastinal lipoma previously. Int J Surg Case Rep 2013;4:302-4. 